\title{
Safe extubation in Obese patient with Totaltrack VLM
}

E. Martinez-Hurtado 1, M. Sanchez-Merchante 2, M. Mariscal Flores 3, J. Ripolles-Melchor 1, N. Aracil Escoda 1, A. Abad Gurumeta1, AnestesiaR alrway Review Group (AiR Group)

1 Consultant Anaesthetist in Infanta Leonor University Hospital. Anesthesia and Intensive care department, Madrid. España.

2 Consultant Anaesthetist in Alcorcon Foundation University Hospital. Anesthesia and Intensive care department, Madrid. Spain.

3 Consultant Anaesthetist in Getafe University Hospital. Anesthesia and Intensive care department. Madrid. Spain

\section{Background}

Extubation may cause laryngospasm, bronchospasm, and elevated intracranial pressure in predisposed subjects (1). Nevertheless, extubation is often considered only a simple "reversal" of intubation. According to data from the NAP4 audit, serious complications during extubation following anesthesia occurred in $13 \%$ of cases and a $5 \%$ mortality was evidenced (2).

\section{Case Report}

We present a 41-year-old male, ASA 2, 35.65 BMI, smoker of 20 cigarettes/day,

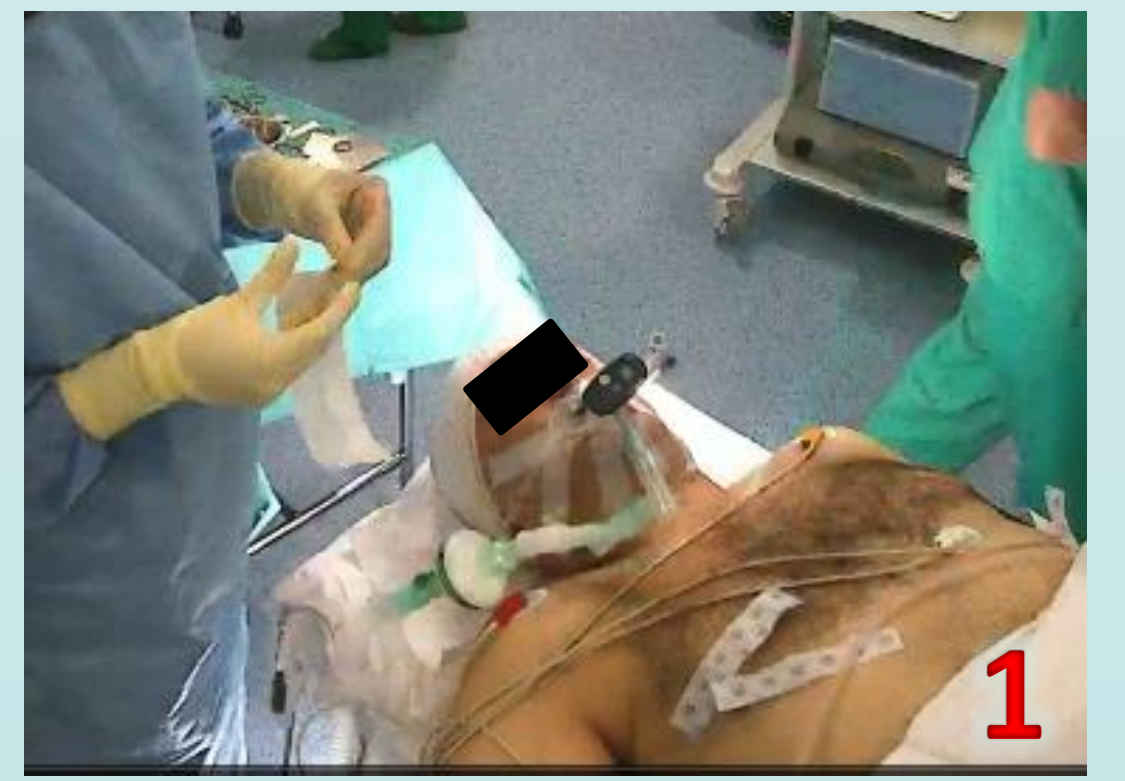
not known allergies, scheduled for left cholesteatoma surgery. Mallampati 3, cervical mobility 90-100, mouth opening 2 , thyromental distance of $6 \mathrm{~cm}$. approx., and upper lip bite test 1.

Intubation was performed with Totaltrack Video Laryngeal Mask (TT VLM) (Medcomflow, Spain) no 4, no 8 flexometallic tube $(E T T)$, on the first attempt, and confirmed with capnography. We extract Videotrack (camera) and rigid blade from VLM Pack, and deinflatable cuff of the flexible blade.

After five hours we perform a safe extubation in a TWO-PHASE extubation process with TT VLM in a Supraglottic Airway Device $(S A D)$ mode (3) with the objective of a smooth eduction, without hemodynamic alterations or respiratory difficulty, and recover of spontaneous ventilation.

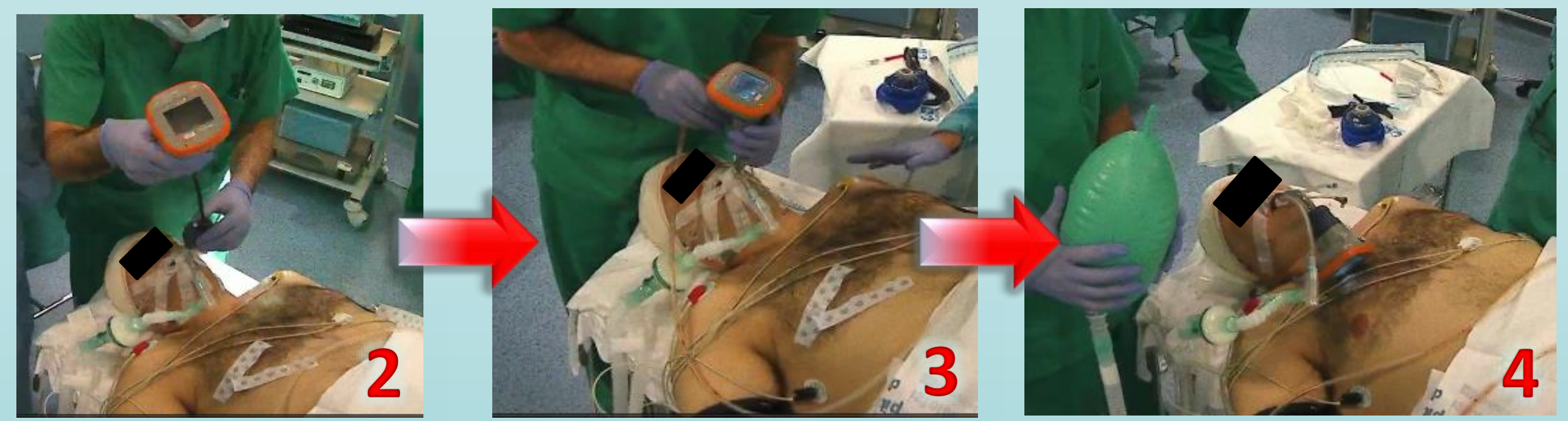

We reconnect Videotrack to assess the larynx, reinflated the SAD cuff, and removed the ETT under direct vision.

When the patient regained spontaneous ventilation, the TT VLM was withdrawn and patient was transferred to reanimation room with supplemental oxygen.

\section{Discussion}

Patients with $\mathrm{BMI}>30$ have low residual volume, low functional residual capacity and poorer response to apnea, OSA (up to 71\%), increased risk of airway obstruction, and rapid oxygen desaturation after anesthesia induction.

Several techniques have been described within an extubation strategy. TT VLM allows a monitored extubation over two phases, going from intubation to supraglottic ventilation mode, and monitoring extubation under continuous viewing or continue the awakening by SAD means.
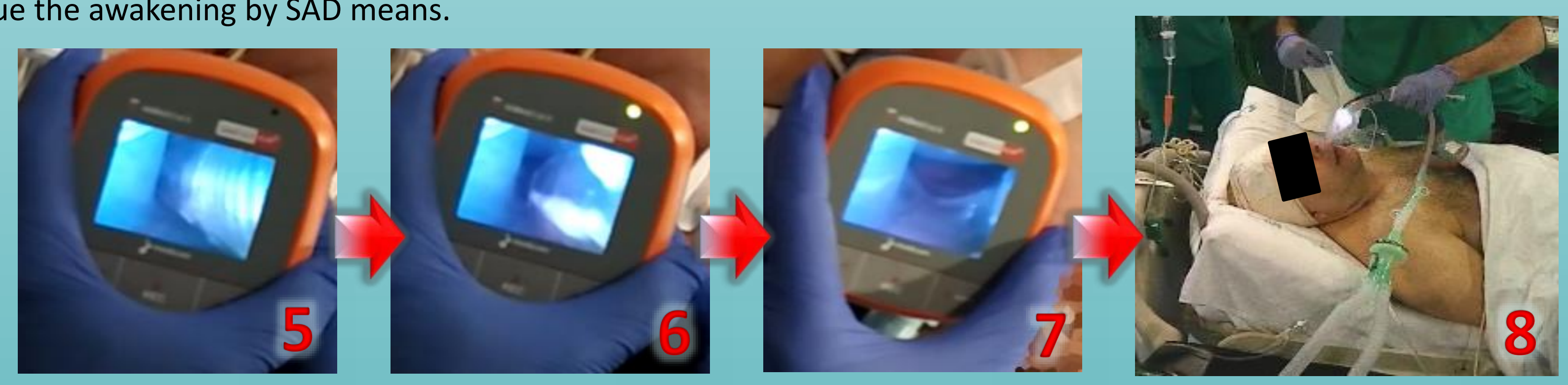

Learning points: Extubation is an elective procedure and must be a well-planned, ensuring the minimum interruption in the administration of oxygen.

\section{References}

1. Asai T. et al. Respiratory complications associated with tracheal intubation and extubation. Br J Anaesth 1998;80:767-75.

2. Cook TM. et al. Fourth National Audit Project. Part 1: anaesthesia. Br J Anaesth. 2011 May;106(5):617-31.

3. Hurtado EM, Merchante MS. Safe Extubation with Totaltrack ${ }^{\circledR}$ VLM. Acad. Anesthesiol. Int. 2016;1(1):3-5. 